\title{
The Milli-Motein: A Self-Folding Chain of Programmable Matter with a One Centimeter Module Pitch
}

\author{
Ara N. Knaian, Kenneth C. Cheung, Maxim B. Lobovsky, \\ Asa J. Oines, Peter Schmidt-Neilsen, and Neil A. Gershenfeld
}

\begin{abstract}
The Milli-Motein (Millimeter-Scale Motorized Protein) is a chain of programmable matter with a $1 \mathrm{~cm}$ pitch. It can fold itself into digitized approximations of arbitrary threedimensional shapes. The small size of the Milli-Motein segments is enabled by the use of our new electropermanent wobble stepper motors, described in this paper, and by a highly integrated electronic and mechanical design. The chain is an interlocked series of connected motor rotors and stators, wrapped with a continuous flex circuit to provide communications, control, and power transmission capabilities. The Milli-Motein uses off-theshelf electronic components and fasteners, and custom parts fabricated by conventional and electric discharge machining, assembled with screws, glue, and solder using tweezers under a microscope. We perform shape reconfiguration experiments using a four-segment Milli-Motein. It can switch from a straight line to a prescribed shape in 5 seconds, consuming $2.6 \mathrm{~W}$ power during reconfiguration. It can hold its shape indefinitely without power. During reconfiguration, a segment can lift the weight of one but not two segments as a horizontal cantilever.
\end{abstract}

\section{INTRODUCTION}

Programmable matter is a universal object or material which is able to change its shape or other physical properties on command. In the quest to realize programmable matter in the lab, much recent effort has focused on the development and miniaturization of electromechanical systems for controlled shape reconfiguration. [8] These systems operate on principles as diverse as hydrodynamic attraction of cubes using controlled flows [11], flexible circuitry able to self-fold itself into oragami using embedded SMA wires [9], magnetic cubes able to self-disassemble on a lattice [7], and cylinders able to active roll over one other using electrostatic forces. [13] The Milli-Motein is a chain of programmable matter, made of interlocked motor rotors and stators, able to fold itself into a digitized representation of an arbitrary threedimensional shape. The mechanical chain is wrapped with a continuous flex circuit to provide communications, control, and power transmission capabilities. The Milli-Motein's small one centimeter module pitch is enabled by a highly integrated mechanical and electronic design, and by the use of our new electropermanent wobble stepper motor. This motor is similar to a conventional electromagnetic wobble motor [3], but controls the flow of magnetic flux using electropermanent magnets [1], [17], [14], [16] rather than electromagnets. As a result, the motor can hold position without power, has enough torque to be used for direct drive without gearing in the Milli-Motein, and is scalable to small sizes without loss of efficiency at low speeds.

The authors are with the Center for Bits and Atoms, Massachusetts Institute of Technology, Cambridge, MA 02139, USA

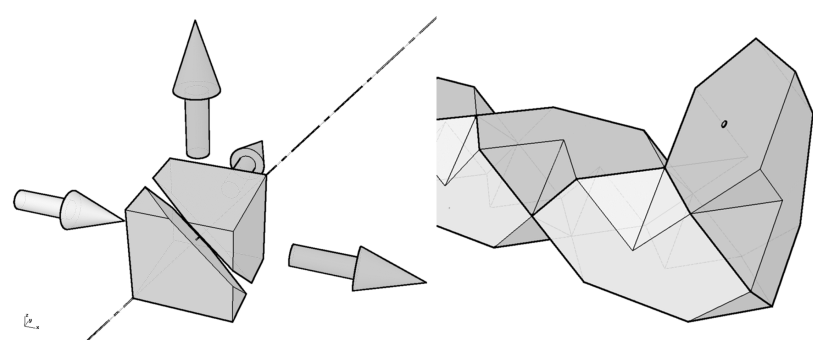

Fig. 1. Hexagonally bisected cube (Molecube) geometry, chained as Cmotein. The Milli-Motein chain has this geometry, to allow it to form digitized approximations of arbitrary geometric shapes.

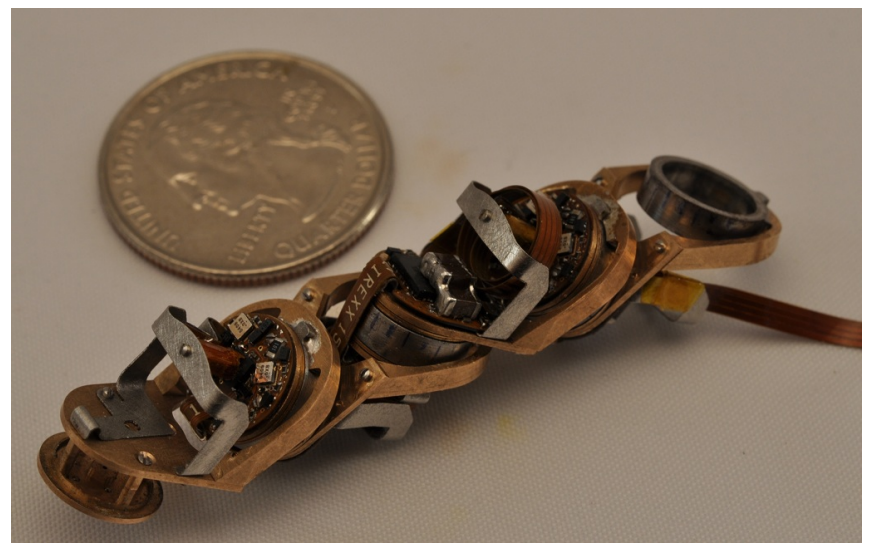

Fig. 2. Four segment Milli-Motein chain with a one centimeter module pitch. The chain folds itself into shapes using our new Electropermanent wobble motors.

\section{GEOMETRY DESIGN}

\section{A. Kinematics}

This section describes the geometric design of the Motein chain which allows it to fold into digitized approximations of arbitrary three-dimensional shapes. Prior work has shown that a small and discrete set of polygonal [5] and polyhedral [4] components can programmatically generate any continuous area and volume filling structure. This provides a strategy for making programmable matter in the form of shape-universal strings. Given typical lattice geometries and a strategy for generating space filling curves [2], many existing reconfigurable robotic systems, can be adapted to chained formats [20], [18], with the advantage of reduced required degrees of freedom per unit, a low number of states per unit, and a power, structural, and communications backbone. It is noted that non Euclidean space tilings are 


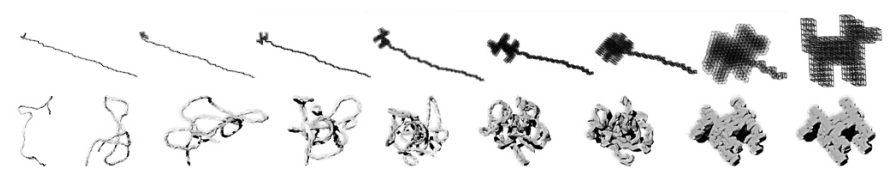

Fig. 3. Serial and parallel motion planning schemes

just as suited these algorithms.

The design of this Motein packs on a simple cubic lattice (C-Motein) in any of its (upper bound $3 \mathrm{O}(\mathrm{n})$, for $\mathrm{n}$ length string) [2] configurations. Each rigid unit along the string is essentially comprised of two halves of adjacent cubes in this final lattice. These halves are formed from the regular hexagonal bisection of the cube. Each unit rotates about the central axis that is orthogonal to this bisection plane, and with respect to the previous adjacent unit along the string. This hexagonally bisected cube kinematic geometry, shown in Figure 1, was first shown in reconfigurable robotics with the Molecube system [20], with reconfigurable connections on cubic modules. In a chain configuration, this is also similar to the Rubik's Snake toy, but with modules that closely pack on a cubic lattice and accordingly with a larger dihedral angle between bearing faces.

\section{B. Motion Planning}

Prior work has begun to explore the efficiency of reverse kinematics and probabilistic roadmap methods for motion planning of very long strings, in order to form goal shapes. We do not yet know if there exists a method to obtain a simultaneous parallel folding for almost every shape. If this is possible, then motion planning for shape making is simple: distribute the folding instruction to all modules and allow all modules to actuate simultaneously with even power distribution. Due primarily to inertial factors at these scales, the effect that we observe in simulations [2] is delayed folding in the middle or anchored end of the string, as the modules towards the end(s) complete their configurations first. A method that we have found for finding a folding strategy for any shape is to simulate the unfolding of the string from the folded state, with collision detection, then to replay the servo positions in reverse order.

As such, for universal shape making, our workflow starts with any common three dimensional CAD file (i.e. binary stereolithography file, describing a three dimensional mesh as a collection of vector facets) evaluated over a lattice. Then, the spanning graph and space filling curve is constructed using this lattice, which is processed into the code for the string. A key aspect of the system is that each unit solves for a local solution. The global solution is a product of the aggregated local results of the programs of each unit. The resulting program is as simple as: turn left or right until you touch your previous neighbor, for each unit.

\section{ELECTROPERMANENT WOBBLE MOTOR}

Figure 4 shows the construction of the electropermanent wobble motor used to actuate the joints of the Milli-Motein.
[15] The stator is cross-shaped, with an outer circular profile. The rotor has a slightly larger inner diameter, and rolls around the stator in an eccentric pattern combining oscillating translation and continuous rotation. [3] Unlike previous magnetic wobble motors, each arm of the stator contains an electropermanent magnet, which is able to be switched on or off with a pulse of current and then retain its magnetization indefinitely. [1], [17], [14] This gives the motor the ability to hold position without power, and improves its efficiency at low speeds.

The rotor, stator core, and stator tips are made from soft magnetic iron. Alnico and $\mathrm{NdFeB}$ permanent magnets are placed in parallel at the center of each arm of the stator to form the electropermanent magnets. An insulated copper wire coil is wrapped around each arm, which is used to switch the magnetization of its Alnico magnet. The NdFeB magnet in each arm has a high coercivity, so is magnetization is not substantially changed by current through the coils. When the Alnico and $\mathrm{NdFeB}$ magnet are magnetized in the same direction, magnetic flux flows through the arm and we say that the arm is switched on. When the Alnico and NdFeB magnet are magnetized in opposite directions, magnetic flux circulates inside the arm and we say that the arm is switched off.

Figure 4 shows how the motor takes a step. In the initial condition, two adjacent arms are switched on, causing the rotor to adhere to their ends by friction. A pulse simultaneously turns off one arm and turns on the opposing arm. The rotor rolls along the perpendicular arm, which stays on continuously, moving away from the arm that switched off and toward the arm that switched on. Repetition of this sequence results in rotation. The $\mathrm{NdFeB}$ permanent magnets are placed with alternating magnetization direction around the stator, so that when adjacent arms are switched on, there can be a closed flux loop through the two arms and the rotor. The magnetic flux paths during operation are shown in Figure 4. Because the coils on opposite arms are always used together, they are continuously wound, so that the device has two electrical phases.

In a conventional electric motor, continuous current (and thus continuous ohmic losses) are required for continuous torque. But in the electropermanent motor, a short pulse of current stores energy in the permanent magnet, and then that energy is converted to mechanical work over the natural mechanical time scale of the system. This allows for continuous holding without power, but it also makes the motor retain efficiency at low speeds, allowing operation at high torque and low power.

\section{HARDWARE DESIGN}

Figure 7 is a photo of an assembled Milli-Motein chain, Figure 6 shows the repeated unit, and Figure 5 is an exploded view diagram of the repeated unit.

\section{A. Electronics}

The flex circuit that wraps around the chain has two alternating circuit boards, so that the motor for each module 


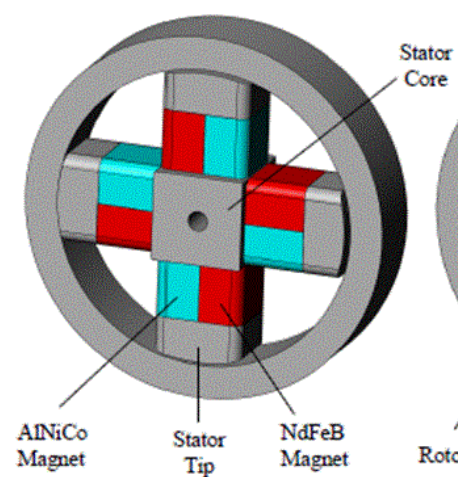

(a)

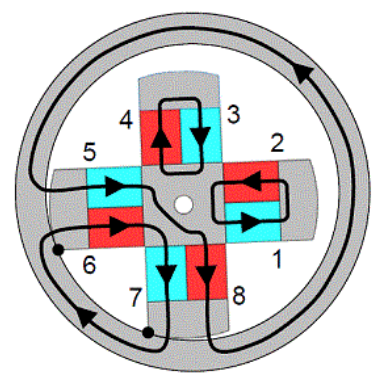

(c)

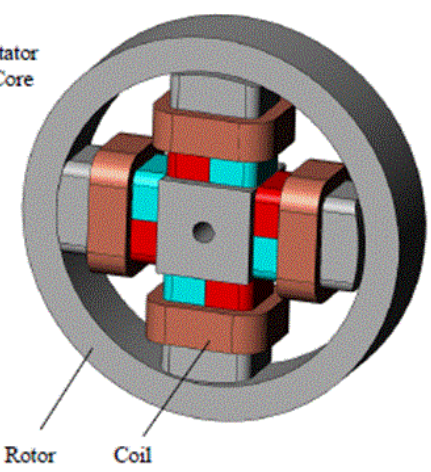

(b)

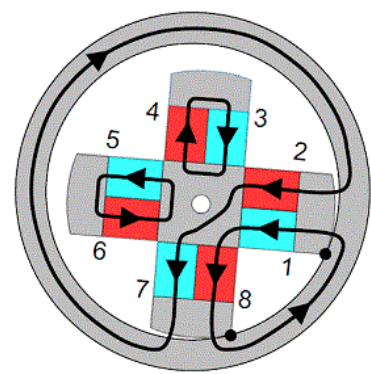

(d)

Fig. 4. Electropermanent Wobble Stepper Motor. Electropermanent stepper motor principle of operation. The red magnets are $\mathrm{NdFeB}$, always polarized in the direction shown. The magnetization of the blue Alnico magnets switches as the motor operates. The motor starts in position (c), with magnets 5-6 and 7-8 on, and magnets 1-2 and 3-4 off. Flux flows through the rotor and stator as shown. Applying a current pulse to the horizontal winding around magnets 5-6 and 2-1 switches the magnetization of magnets 5 and 1, turning magnet 5-6 off and magnet 2-1 on, resulting in the new flux paths shown at right. The rotor pivots counterclockwise about magnet 7-8, taking one step to arrive at the new position shown in (d). Repeated steps drives the rotor abound the stator with a continuous rotary motion and oscillating translational motion.

is sandwiched between one board of each type. The processor board contains an 8-bit microcontroller, a bank of capacitors to store the energy required for one motor pulse, and one of three half-bridge motor drivers. The motor driver board contains the other two half-bridge motor drivers, as well as the two-axis magnetoresistive position sensor. Each processor has two serial data communication ports, which it uses to communicate with the two adjacent processors on the chain. $\mathrm{DC}$ power is bussed along the chain at $5 \mathrm{~V}$ for the electronics and $29 \mathrm{~V}$ for the motors.

\section{B. Mechanics}

Each module is built around an electropermanent stepper motor. The magnetic materials for the stator (Iron, Alnico, and $\mathrm{NdFeB}$ ) are cut to size by wire EDM, cleaned, and glued together with epoxy. We then wind a coil around each arm of the stator, and set the bond-coat of the wire with heat to keep the coil from unraveling. When the chain is assembled the rotor from the next unit goes around the stator as shown in Figure 7 . The rotor is an iron ring, made by milling. The rotor

inner diameter is 75 micron larger than the outer diameter to permit the wobble motor action.

The rotor is constrained axially by two ball thrust bearings, which are sandwiched between the motor-driver-side and processor-side bushings. They allow the rotor to freely rotate, and to move radially and tangentially over a limited range, but do not allow axial motion. The ball thrust bearings consist of eight 500 micron diameter stainless steel balls, held in an evenly-spaced circular pattern by a bronze ball retainer. The top and bottom halves of the ball retainers are made by milling, the balls are inserted with tweezers, and then the ball retainers are soldered together to retain the balls.

The motor-driver-side and processor-side bushings are major structural components of the module. The electropermanent motor stator core is fastened to a pedestal at the center of the motor-driver-side bushing using a screw. The motor wires are routed through holes in the bushing, surrounded by 500 micron diameter medical Teflon tubing to protect them from damage. After the rotor and ball thrust bearings are inserted over the stator, the processor bushing is screwed on to the other side, forming a closed package. The motor-driver bushing and processor-bushing have integrated standoffs so that they can attach firmly to one another and leave about 50 micron of axial play in the rotor when the stack is assembled. The flex circuit assembly attaches with screws to the bushings on each side of the motors. The motor wires protrude through un-plated holes on the motor driver board and are soldered to adjacent SMT pads.

Each module connects to the next module though the rotor joiner, a bracket that screws to the processor bushing of one module and is soldered to the rotor of the next module. The rotor joiner creates the 109.57 degree angle between adjacent modules that is required for the hexagonally-bisected cube geometry.

A coiled section in the flex circuit allows it to transition between modules. As the modules rotate, the coil expands and contracts. The cable carrier and cable cover are made from 125 micron-thick folded stainless steel. They act to constrain the flex circuit so that is moves in the intended way. The cable carrier holds a flex circuit tab upright near the center of the module, to keep the coil from falling over. The cable cover keeps the coil constrained axially, to prevent tangling. A cylindrical permanent magnet is mounted from the cable cover, so that it sits above the two-axis magnetoresistive sensor of the adjacent module. Together, they form a rotary position sensor that allows the module to measure its angle with respect to the previous module, to allow for position control and shape reconfiguration.

\section{Software}

Each processor runs identical firmware, a command monitor that processes commands and issues responses. A program running on a PC provides high-level control of shape reconfiguration, sending commands down the serial chain connecting the processors.

Upon power-up, the PC sends the discover command, which is passed down the chain with an incrementing 

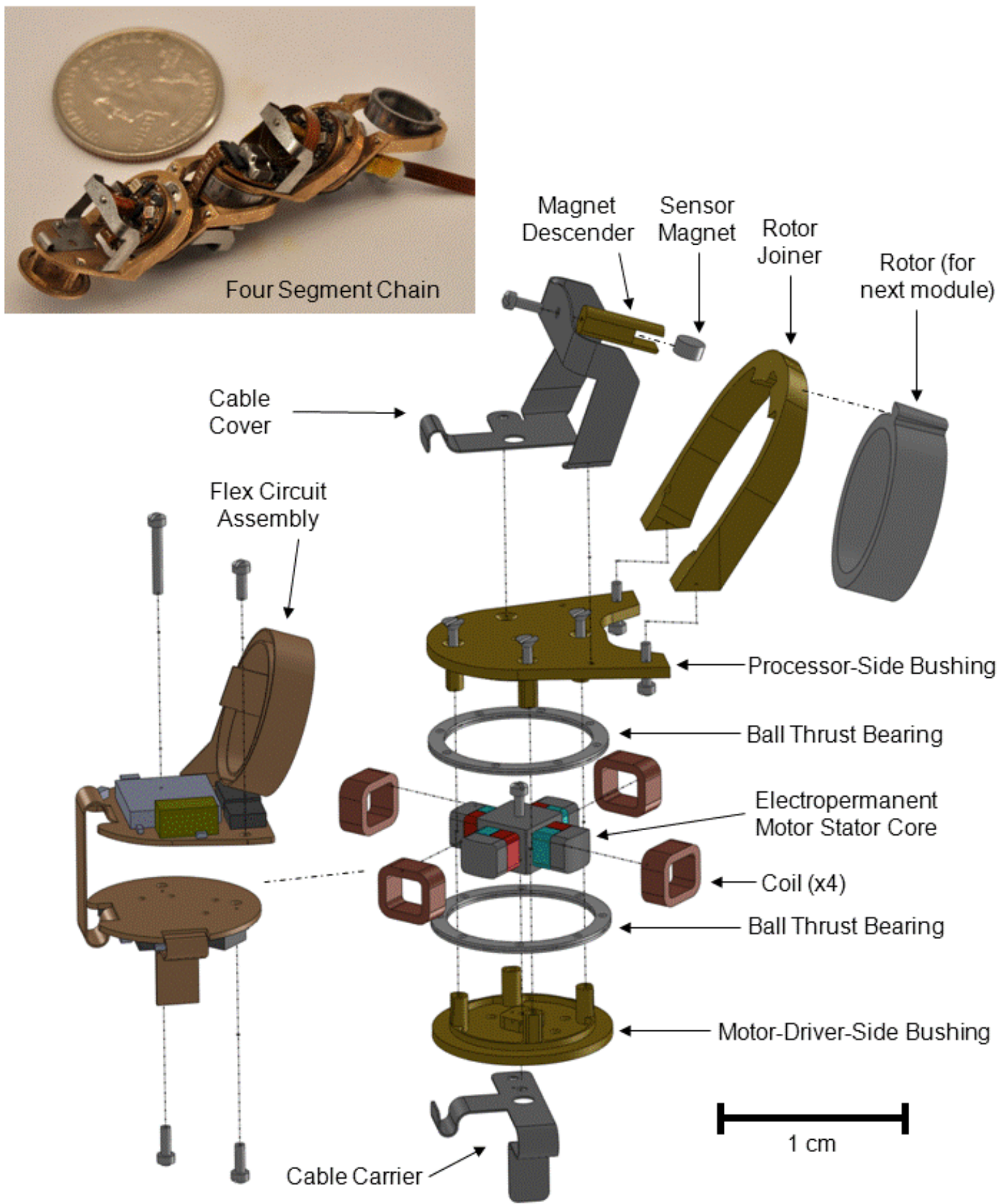

Fig. 5. This assembly is repeated for each joint. One segment of the continuous flex circuit is shown in its folded configuration. The stator on this module mates with the rotor on the adjacent module. See Figure 6 for an assembled view. 


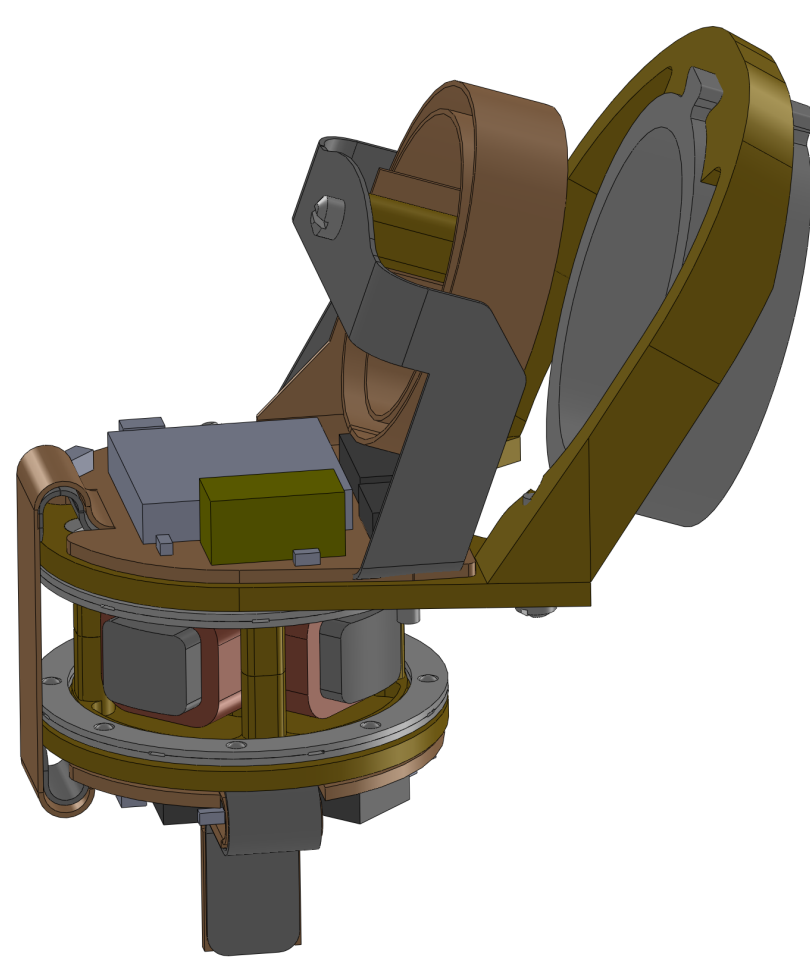

Fig. 6. This assembly is repeated for each joint. The stator on this module mates with the rotor on the adjacent module. See Figure 5 for an exploded view.

number, allowing each chain to discover its ID. Thereafter, the PC can select individual nodes and read or write their registers. In doing so, the PC can read the current angle, set a desired angle, and initiate motion. The geometry of the chain is fully described by its vector of joint angles. The output of the folding workflow is a sequence of joint angle vectors that define a path through configuration space for the chain to take to reconfigure from one shape to another. During shape reconfiguration, the PC loads each node with its desired angle, initiates motion of all nodes simultaneously, waits for completion, and then repeats for each step in the reconfiguration sequence, until the desired endpoint geometry is achieved.

\section{RESULTS}

\section{A. Assembly}

Assembling the Milli-Motein proved to be a very challenging manual operation. We manipulated the parts using titanium tweezers under a stereo microscope, and joined them by soldering, using epoxy, or fastening using \#0000160 (500 micron diameter) screws. In many ways, our approach to assembly was to extend the family of techniques used for electronics assembly at this scale to assemble the mechanical parts of the system as well. We used soldering to join metal parts, epoxy to join the heat-sensitive permanent magnets, and screws to reversibly fasten subassemblies that might require subsequent removal for repair. Due to the small

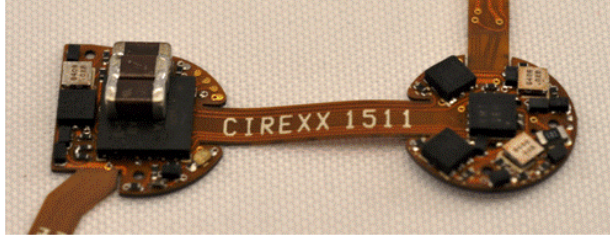

(a)

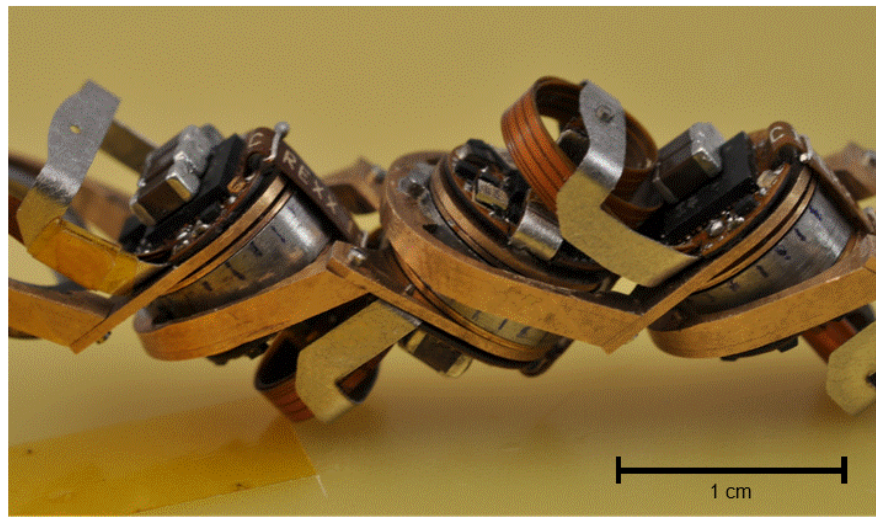

(c)

Fig. 7. Milli-Motein Detail Views. (a) The repeating section of the MilliMotein flex circuit. The processor board (left) contains an 8-bit microcontroller, a capacitor bank to store pulse power for the actuator, and one of the three half-bridge motor drivers. The motor driver board (right) contains the other two half-bridge motor drivers and two-axis magnetoresistive sensor at the center. (b) Partially assembled electropermanent motor. Only one the four coils are installed in this photo. The stator core and the tips of the stator arms are made of iron. Each arm is interrupted by a parallel pair of $\mathrm{NdFeB}$ and Alnico magnets, forming a switchable electropermanent magnet. A ball thrust bearing and motor-driver-side bushing are also visible in this photo (c) Fully assembled Milli-Motein.

size of the parts, a significant portion of the time for assembly was devoted to scraping and cleaning procedures, to remove grease, waxy deposits, fibrous materials, corrosion, dirt, and EDM scale from the parts to prepare them for assembly.

The small size of the parts relative to our tools made them fragile - not during actual operation of the robotic system — but during assembly and especially repair. During assembly, ripping or kinking of the flex circuit, failure of epoxied joints, nicking of magnet wire insulation, knocking components off the board, and stripping of threaded fasteners were not uncommon occurrences. To compound matters, a relatively small mechanical defect (such as a nicked wire) could then lead to a serious and systemic electrical fault requiring major repair work that could then lead to additional mechanical damage. Through design revisions to facilitate assembly, carefully planning and documenting the assembly procedure, and steadily increasing mechanical skill, we were finally able to construct a working four-module prototype.

\section{B. Shape Reconfiguration Testing}

We placed a four-segment Milli-Motein on an FR-4 platform. We controlled the system using a PC connected to the first node in the chain with a serial link, and supplied power using a bench supply. The operator could command the Motein to change between the four shapes shown in 


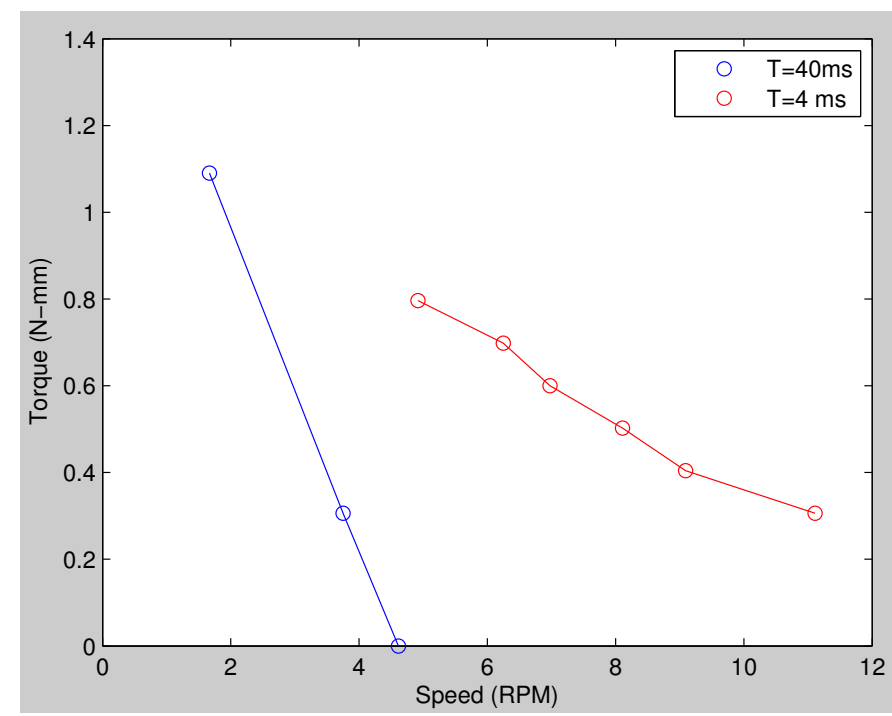

Fig. 8. Electropermanent stepper torque vs. speed curves, measured by hanging weights on a string from the rotor, and measuring the time for one revolution with a stopwatch. $\mathrm{T}$ is the time between switching pulses.

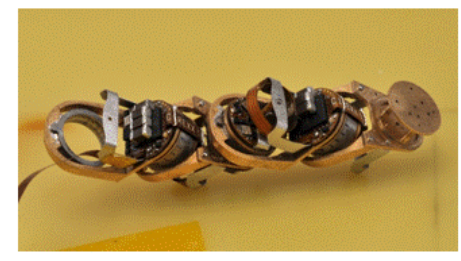

(a)

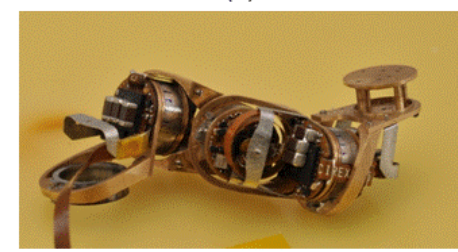

(c)

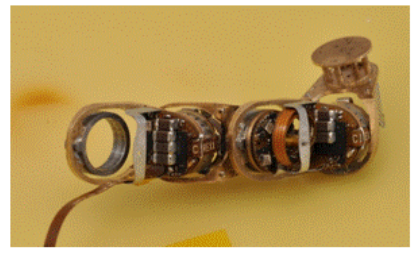

(b)

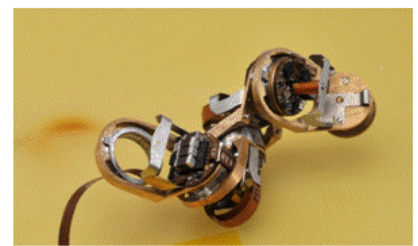

(d)
Fig. 9. Four-segment Milli-Motein rendering four geometric shapes. (a) straight line [0 00 0] (b) L-shape [0 00 90] ( c ) periscope [-90 0 90] (d) helix. [90 90 90]

\section{Figure 9.}

The chain drew $35 \mathrm{~mA}$ on the $5 \mathrm{~V}$ rail continuously, to power the processor and sensors, and about $100 \mathrm{~mA}$ on the $29 \mathrm{~V}$ rail during shape reconfiguration, to power three motors operating simultaneously. Shape reconfiguration time through 90 degrees was about 5 seconds. The motor step pulse length was $60 \mu \mathrm{s}$, and the pulse-to-pulse time was 10 ms, with eight pulses per step cycle.

The mass of each segment was 3.5 grams. With the chain extended in a straight horizontal line, a single node was able to hold the weight of the other three nodes without its rotor slipping. Nodes were able to actively lift a one-node cantilever through the maximum-torque horizontal position, but not able to lift a two-node cantilever. (Attempting to lift a two-node cantilever resulted in very slow backward motion.)

The distance from the center of rotation of the motor to the center of mass of the node it lifts is $13 \mathrm{~mm}$. Based on this, it should take $0.45 \mathrm{~N}$-mm torque to lift a one-node cantilever, and $1.34 \mathrm{~N}-\mathrm{mm}$ torque to lift a two-node cantilever. From Figure 8, the maximum forward torque of the motor is between these two figures, so this is a consistent result. The Milli-Motein was able to move itself around on the table, so it seems likely that a battery-powered Milli-Motein could move itself across a table. The Milli-Motein was also able to reconfigure from a stable shape to an unstable shape, which would then dramatically fall over; this is shown in the video clip. The wobble motors made a clicking noise during operation, resembling the sound of a film movie projector.

It was possible to switch the chain from a straight line to any of the four shapes shown above and back to a straight line simply by commanding all nodes to go to the desired angle at once, without any motion planning. However, even with this fairly short chain, motion planning would have allowed for a greater variety of shapes to be formed. Sequencing of the moves to lift up certain parts of the chain before others, it was possible to reach configurations that could not be reached by simply driving all actuators toward the target at once, because in this case the chain would wedge itself against the table. The same configuration (e.g. a straight line) has multiple stable orientations on the table, so some information about the overall orientation of the chain (perhaps from a single two-axis accelerometer at one end of the chain) would be needed in order to do gravity and motor torque-aware motion planning.

\section{DISCUSSION OF SCALABILITY}

If all of the linear dimensions of the chain were to be divided by a constant factor, what techniques could we use to fabricate it, and then, once assembled, would it work? There are a number of factors to consider; some fundamental and some practical.

\section{A. Scaling of Force and Energy}

The torque required to lift a cantilever of a fixed number of nodes scales with the fourth power of length. The shaft power required to do so at a given angular speed also scales with the fourth power of length. The torque of the motors is limited by magnetic saturation, with the maximum force proportional to the pole area, and thus the maximum torque proportional to the cube of length. Since the resisting force goes with the fourth power but the maximum torque with the third, we could expect each node to be able to lift a greater number of its neighbors in a cantilever the system dimensions are scaled down.

The energy dissipated by the electropermanent motor in hysteresis and ohmic losses is proportional to the work done [14], and so we can expect the total power required by motors to scale with the fourth power of length. Given a power source of constant volumetric energy density, then, we could expect proportionally less drain on the batteries as the system is scaled down, to execute identical motions at an identical angular speed on the scaled-down system. 
As the system is scaled down, at some point, forces other than node weight will become the dominant resisting force. The motor torque is scalable against forces proportional to area, but not against forces proportional to length.

\section{B. Scaling of Fabrication}

This system uses the smallest size of off-the-shelf electronic components, so a custom IC (perhaps with custom packaging) would be required to miniaturize it much further. The coils were wound with 36 gauge wire. Magnet wire is readily available down to 60 gauge, which is 16 times smaller. The machining tolerance for the motor was 25 microns, within the range of conventional machining. The motor parts (and their machining tolerance) could be scaled down by a factor of 10 and still be within the tolerance range for wire EDM machining, However, below this, alternative fabrication techniques (e.g. LIGA) would be needed.

Assembly of the system with tweezers by hand proved challenging. With improved mechanical design and fixtures, it might be possible to build modules a few times smaller. But we expect that a parallel-batch automated assembly process will ultimately allow construction of higher-resolution systems at reasonable cost.

\section{CONCLUSION}

The Milli-Motein is functional as programmable matter, able to reconfigure itself into several shapes on command. As far as we know, it is the highest-resolution chain-type programmable matter system built to date. Yet clearly it is a long way from something that would find widespread application as a universal material. That material will need to be much cheaper, stronger, less fragile, and will need to have many more degrees of freedom. Additionally, scaling to large numbers of nodes will require improved software and algorithms to make the system decentralized and faulttolerant. Programmable matter could be produced using existing MEMS techniques, [12] but currently available processes place great restrictions on the materials and geometries that can be employed. Development of improved technologies for three-dimensional free-form fabrication of miniaturized electromechanical systems, automated systems for high-speed robotic assembly of micro-parts [6], [10], or roll-to-roll lamination of patterned layers [19] are in our view the next logical steps in the quest to cost-reduce and miniaturize robotic systems to the point that they could truly be thought of as materials.

\section{ACKNOWLEDGMENTS}

This work is supported by the DARPA Programmable Matter and M3 programs (U.S. Army Research Office grants W911NF-08-1-0254, W911NF-11-1-0096), and the MIT Center for Bits and Atoms. We thank Daniela Rus, Markus Zahn, Joe Jacobson, Robert Wood, and Paul Lituri for their advice, and thank Amy Sun and Nadya Peek for their help with photography.

\section{REFERENCES}

[1] Philibert Maurice Braillon. Magnetic plate comprising permanent magnets and electropermanent magnets. US Patent 4,075,589.

[2] K.C. Cheung, E.D. Demaine, J.R. Bachrach, and S. Griffith. Programmable assembly with universally foldable strings (moteins). Robotics, IEEE Transactions on, 27(4):718 -729, aug. 2011.

[3] P. Dario, M.C. Carrozza, C. Stefanini, and S. D'Attanasio. A mobile microrobot actuated by a new electromagnetic wobble micromotor. Mechatronics, IEEE/ASME Transactions on, 3(1):9-16, March 1998.

[4] Erik Demaine, Martin Demaine, Jeffrey Lindy, and Diane Souvaine. Hinged dissection of polypolyhedra. In Frank Dehne, Alejandro LpezOrtiz, and Jrg-Rdiger Sack, editors, Algorithms and Data Structures, volume 3608 of Lecture Notes in Computer Science, pages 205-217. Springer Berlin / Heidelberg, 2005.

[5] Erik D. Demaine, Martin L. Demaine, David Eppstein, Greg N. Frederickson, and Erich Friedman. Hinged dissection of polyominoes and polyforms. Computational Geometry, 31(3):237 - 262, 2005. 11th Canadian Conference on Computational Geometry.

[6] N. A. Gershenfeld. The Coming Revolution on Your Desktop - From Personal Computers to Personal Fabrication. Basic Books, New York, NY, 2005.

[7] K. Gilpin, A. Knaian, and D. Rus. Robot pebbles: One centimeter modules for programmable matter through self-disassembly. In Robotics and Automation, 2010. ICRA '10. IEEE International Conference on, Anchorage, AK, 2010. IEEE.

[8] K. Gilpin and D. Rus. Modular robot systems. Robotics Automation Magazine, IEEE, 17(3):38 -55, sept. 2010.

[9] E. Hawkes, B. An, N. M. Benbernou, H. Tanaka, S. Kim, E. D. Demaine, D. Rus, and R. J. Wood. Programmable matter by folding. Proceedings of the National Academy of Sciences, 107(28):1244112445, 2010.

[10] J.D. Hiller, J. Miller, and H. Lipson. Microbricks for three-dimensional reconfigurable modular microsystems. Microelectromechanical Systems, Journal of, 20(5):1089 -1097, oct. 2011.

[11] Michael Kalontarov, Michael Tolley, Hod Lipson, and David Erickson. Hydrodynamically driven docking of blocks for $3 \mathrm{~d}$ fluidic assembly. Microfluidics and Nanofluidics, 9:551-558, 2010. 10.1007/s10404010-0572-9.

[12] M.E. Karagozler, J.D. Campbell, G.K. Fedder, S.C. Goldstein, M.P. Weller, and B.W. Yoon. Electrostatic latching for inter-module adhesion, power transfer, and communication in modular robots. In Intelligent Robots and Systems, 2007. IROS 2007. IEEE/RSJ International Conference on, pages 2779-2786, October 2007.

[13] Mustafa Emre Karagozler, Seth Copen Goldstein, and J. Robert Reid. Stress-driven MEMS assembly + electrostatic forces $=1 \mathrm{~mm}$ diameter robot. In Proceedings of the IEEE International Conference on Intelligent Robots and Systems (IROS '09), October 2009.

[14] A.N. Knaian. Electropermanent Magnetic Connectors and Actuators: Devices and their Application in Programmable Matter. PhD thesis, Massachusetts Institute of Technology, 2010.

[15] Ara Knaian. Electropermanent magnet-based motors. US Patent Application US2010/0289359 A1.

[16] Andrew D. Marchese, Cagdas D. Onal, and D Rus. Soft robot actuators using energy-efficient valves controlled by electropermanent magnets. Intelligent Robots and Systems, 2000. (IROS 2011). Proceedings. 2011 IEEE/RSJ International Conference on, pages 756-761, 2011.

[17] Dominic F. Pignataro. Electrically switchable magnet system. US Patent 6,229,422.

[18] George M. Whitesides and Mila Boncheva. Beyond molecules: Selfassembly of mesoscopic and macroscopic components. Proceedings of the National Academy of Sciences, 99(8):4769-4774, 2002.

[19] R. J. Wood, S. Avadhanula, R. Sahai, E. Steltz, and R. S. Fearing. Microrobot design using fiber reinforced composites. Journal of Mechanical Design, 130(5):052304, 2008.

[20] V. Zykov, E. Mytilinaios, B. Adams, and H. Lipson. Self-reproducing machines. Nature, 435:163-164, 2005. 